\title{
Light relic neutralinos
}

\author{
A. Bottino, ${ }^{*}$ N. Fornengo, ${ }^{\dagger}$ and S. Scopel ${ }^{\ddagger}$ \\ Dipartimento di Fisica Teorica, Università di Torino, Istituto Nazionale di Fisica Nucleare, Sezione di Torino, via P. Giuria 1, \\ I-10125 Torino, Italy
}

(Received 9 January 2003; published 28 March 2003)

\begin{abstract}
The relic abundance and the scalar cross section off the nucleon for light neutralinos (of mass below about $45 \mathrm{GeV}$ ) are evaluated in an effective MSSM model without GUT-inspired relations among the gaugino masses. It is shown that these neutralinos may provide a sizable contribution to the matter density in the Universe and produce measurable effects in WIMP direct detection experiments. These properties are elucidated in terms of simple analytical arguments.
\end{abstract}

DOI: 10.1103/PhysRevD.67.063519

PACS number(s): 95.35.+d, 11.30.Pb, 12.60.Jv, 95.30.Cq

\section{INTRODUCTION}

Most works on relic neutralinos consider supersymmetric schemes with a unification assumption for the gaugino masses $M_{i}(i=1,2,3)$ at the grand unified theory (GUT) scale $M_{G U T} \sim 10^{16} \mathrm{GeV}$. This hypothesis implies that at lower scales the following relations hold:

$$
M_{1}: M_{2}: M_{3}=\alpha_{1}: \alpha_{2}: \alpha_{3},
$$

where the $\alpha_{i}(i=1,2,3)$ are the coupling constants of the three standard model gauge groups. In particular, at the electroweak scale, $M_{E W} \sim 100 \mathrm{GeV}, M_{1}$ and $M_{2}$ are related by the expression

$$
M_{1}=\frac{5}{3} \tan ^{2} \theta_{W} M_{2} \simeq 0.5 M_{2} .
$$

However, there are theoretical arguments for considering supersymmetric schemes where the unification assumption on gaugino masses is not satisfied [1].

In the present paper we analyze the properties of relic neutralinos in an effective minimal supersymmetric extension of the standard model (MSSM) where the GUT relation of Eq. (2) is relaxed. Previous papers where supersymmetric schemes without gaugino mass unification have been considered in connection with relic neutralinos include the ones reported in Refs. [2-13]. Here we evaluate the neutralino relic abundance $\Omega_{\chi} h^{2}$ and the neutralino-nucleon scalar cross section $\sigma_{\text {scalar }}^{\text {(nucle })}$, which is relevant to dark matter direct detection. In Sec. II we define the supersymmetric scheme adopted in the present paper and in Sec. III we provide analytical considerations and numerical evaluations. Our conclusions are drawn in Sec. IV.

\section{EFFECTIVE MSSM WITHOUT GAUGINO UNIFICATION}

We employ an effective MSSM scheme (EMSSM) at the electroweak scale, defined in terms of a minimal number of

\footnotetext{
*Electronic address: bottino@to.infn.it

${ }^{\dagger}$ Electronic address: fornengo@to.infn.it

*Electronic address: scopel@to.infn.it
}

parameters, only those necessary to shape the essentials of the theoretical structure of MSSM and of its particle content. The assumptions that we impose at the electroweak scale are (a) all squark soft-mass parameters are taken degenerate: $m_{\tilde{q}_{i}} \equiv m_{\tilde{q}}$; (b) all slepton soft-mass parameters are taken degenerate: $m_{\tilde{l}_{i}} \equiv m_{\tilde{l}}$; (c) all trilinear parameters are set to zero except those of the third family, which are defined in terms of a common dimensionless parameter $A: A_{\tilde{b}}=A_{\tilde{t}} \equiv A m_{\tilde{q}}$ and $A \tilde{\tau} \equiv A m_{\tilde{l}}$. As a consequence, the supersymmetric parameter space consists of the following independent parameters: $M_{2}, \mu, \tan \beta, m_{A}, m_{\tilde{q}}, m_{\tilde{l}}, A$, and $R \equiv M_{1} / M_{2}$. In the previous list of parameters we have denoted by $\mu$ the Higgs mixing mass parameter, by $\tan \beta$ the ratio of the two Higgs vacuum expectation values (VEVs) and by $m_{A}$ the mass of the $C P$-odd neutral Higgs boson.

This scheme differs from the EMSSM which we employed for instance in Ref. [14] in the fact that we are relaxing here the gaugino unification relation, which was instead assumed in our previous works. The presence of the extra $R$ parameter accounts for this fact.

The neutralino is defined as the lowest-mass linear superposition of $B$-ino $\widetilde{B}, W$-ino $\widetilde{W}^{(3)}$ and of the two Higgsino states $\widetilde{H}_{1}^{\circ}, \widetilde{H}_{2}^{\circ}$ :

$$
\chi \equiv a_{1} \widetilde{B}+a_{2} \widetilde{W}^{(3)}+a_{3} \widetilde{H}_{1}^{\circ}+a_{4} \widetilde{H}_{2}^{\circ} .
$$

Because of well-known properties of the neutralino and chargino mass matrices, one has that (a) for $\mu \gg M_{1}, M_{2}$ the neutralino mass is determined by the lightest gaugino mass parameter: $m_{\chi} \simeq \min \left(M_{1}, M_{2}\right)$, while the lightest chargino mass is set by $M_{2}: m_{\chi^{ \pm}} \simeq M_{2}\left(M_{1}\right.$ does not enter the chargino mass matrix at the tree level), and (b) for $\mu$ $\ll M_{1}, M_{2}$ both the neutralino and the chargino masses are primarily set by the Higgs mixing parameter: $m_{\chi} \simeq \mu$ $\simeq m_{\chi^{ \pm}}$.

CERN $e^{+} e^{-}$collider LEP data put a stringent lower bound on the chargino mass: $m_{\chi^{ \pm}} \gtrsim 103 \mathrm{GeV}$, which converts into lower bounds on $M_{2}$ and $\mu: M_{2}, \mu \gtrsim 103 \mathrm{GeV}$. This implies a lower bound on the neutralino mass of the order of about $50 \mathrm{GeV}$ in the standard EMSSM, where the GUT relation of Eq. (2) holds. On the contrary, the neutralino mass may be smaller when $M_{1} \ll M_{2}$, thus for small values of the parameter $R$. 
In the present paper we are interested in the phenomenology of light neutralinos, therefore we consider values of $R$ lower than its GUT value: $R_{G U T} \simeq 0.5$. For definiteness we will consider the range $0.01-0.5$. The ensuing light neutralinos have a dominant $B$-ino component; a deviation from a pure $B$-ino composition is mainly due to a mixture of $\widetilde{B}$ with $\widetilde{H}_{1}^{\circ}$, as will be shown in Sec. III B.

In our numerical analysis we have varied the MSSM parameters within the following ranges: $1 \leqslant \tan \beta \leqslant 50$, $100 \mathrm{GeV} \leqslant|\mu|, M_{2}, m_{\tilde{q}}, m_{\tilde{l}} \leqslant 1000 \mathrm{GeV}, \quad \operatorname{sgn}(\mu)=-1,1$, $90 \mathrm{GeV} \leqslant m_{A} \leqslant 1000 \mathrm{GeV},-3 \leqslant A \leqslant 3$, for a sample of representative values of $R$ in the range $0.01 \leqslant R \leqslant 0.5$. This range for $R$, implemented with the experimental lower limit on $M_{2}$ of about $100 \mathrm{GeV}$, implies that the lower bound on the neutralino mass can be moved down to few GeV's for $R$ $\sim 0.01$.

We then implemented the following experimental constraints: accelerators data on supersymmetric and Higgs boson searches [CERN $e^{+} e^{-}$collider LEP2 [15] and Collider Detector at Fermilab (CDF) [16]]; measurements of the $b$ $\rightarrow s+\gamma$ decay [17]. We wish to comment that the accelerator limits on the Higgs sector are taken into account by implementing the limits on the Higgs boson production cross sections: $e^{+} e^{-} \rightarrow h Z$ and $e^{+} e^{-} \rightarrow h A$ ( $h$ and $A$ are the lightest scalar and the pseudoscalar neutral Higgs bosons, respectively), which in turn imply a constraint on the coupling constants $\sin ^{2}(\alpha-\beta)$ and $\cos ^{2}(\alpha-\beta)$. Once these limits are applied, the absolute lower limit on the Higgs boson masses is $m_{A}, m_{h} \sim 90 \mathrm{GeV}$. The allowed light-Higgs boson mass range between 90 and $114 \mathrm{GeV}$ is very often overlooked in studies of neutralino dark matter, where a flat limit of 114 $\mathrm{GeV}$ is applied to $m_{h}$. The light-Higgs boson mass range, even though difficult (but not impossible) to be achieved in supergravity (SUGRA) models [18-20], is nevertheless quite natural in the EMSSM and usually provides large detection rates for neutralino dark matter [19].

As for the constraint due to the muon anomalous magnetic moment $a_{\mu} \equiv\left(g_{\mu}-2\right) / 2$ we have used the interval $-160 \leqslant \Delta a_{\mu} \times 10^{11} \leqslant 680$, where $\Delta a_{\mu}$ is the deviation of the current world average of the experimental determinations (dominated by the measurements of Ref. [21]) from the theoretical evaluation within the standard model: $\Delta a_{\mu} \equiv a_{\mu}^{\text {expt }}$ $-a_{\mu}^{S M}$. The range we use for $\Delta a_{\mu}$ is a $2 \sigma$ interval, obtained by using for the lowest-order hadronic vacuum polarization contribution an average between the results derived from the $e^{+}-e^{-}$data [22,23] and from hadronic $\tau$ decays [22]. The $\Delta a_{\mu}$ constraint and the $b \rightarrow s+\gamma$ bound set stringent limits for the light neutralino sector of our models.

Once also the relic abundance bound $\Omega_{\chi} h^{2} \leqslant 0.3$ is applied (see Sec. III B) in addition to the other experimental constraints discussed above, a lower limit of about $6 \mathrm{GeV}$ is obtained for the neutralino mass in the class of models with nonuniversal gaugino masses considered in this paper [3335].

\section{NEUTRALINO RELIC ABUNDANCE AND NEUTRALINO-NUCLEON CROSS SECTION}

\section{A. Some analytical properties for small $m_{\chi}$}

The neutralino configurations which provide the highest values of direct detection rates are the ones dominated by $(h, H)$ Higgs-exchange processes, which in turn require a gaugino-Higgsino mixing. For these configurations, also the relic abundance is regulated by a $(A)$ Higgs-exchange diagram in the $\chi-\chi$ annihilation cross section.

Thus, to get an insight into the properties to be expected for our light neutralinos we limit ourselves to the following approximate expressions, derived under the assumptions of Higgs dominance and light neutralinos (notice however that full exact expressions both for the relic abundance $\Omega_{\chi} h^{2}$ and for the neutralino-nucleon scalar cross section $\sigma_{\text {scalar }}^{\text {(nucleon) }}$ are employed in the numerical evaluations to be discussed in the next section). Under these hypotheses, the neutralino relic abundance is dominated by the $s$-wave annihilation in a $\bar{b} b$ pair (unless $m_{\chi}$ is very close to the $b$-quark mass $m_{b}$, in which case the $\bar{c} c$ and $\bar{\tau} \tau$ channel are dominant):

$$
\begin{aligned}
\Omega_{\chi} h^{2} \simeq & \frac{4 \times 10^{-39} \mathrm{~cm}^{2}}{\left\langle\sigma_{\mathrm{ann}} v\right\rangle_{\mathrm{int}}} \\
\simeq & \frac{10^{-37} \mathrm{~cm}^{2}}{6 \pi \alpha_{e m}^{2}} \frac{\sin ^{4} \theta_{W}}{\tan ^{2} \beta(1+\epsilon)^{2}} \\
& \times\left(a_{2}-a_{1} \tan \theta_{W}\right)^{-2}\left(a_{4} \cos \beta-a_{3} \sin \beta\right)^{-2} \\
& \times \frac{\left[\left(2 m_{\chi}\right)^{2}-m_{A}^{2}\right]^{2}}{m_{\chi}^{2}\left[1-m_{b}^{2} / m_{\chi}^{2}\right]^{1 / 2}} \frac{m_{W}^{2}}{m_{b}^{2}},
\end{aligned}
$$

and the elastic scattering cross section is

$$
\sigma_{\text {scalar }}^{\text {(nucleon) }} \simeq \frac{8 G_{F}^{2}}{\pi} M_{Z}^{2} m_{\text {red }}^{2}\left[\frac{F_{h} I_{h}}{m_{h}^{2}}+\frac{F_{H} I_{H}}{m_{H}^{2}}\right]^{2} .
$$

In the previous equations we have used the following notations: $\left\langle\sigma_{\text {ann }} v\right\rangle_{\text {int }}$ is the integral from present temperature up to the freeze-out temperature of the thermally averaged product of the annihilation cross section times the relative velocity of a pair of neutralinos; $\epsilon$ is a quantity which enters in the relationship between the down-type fermion running masses and the corresponding Yukawa couplings (see, for instance, Refs. [18,25] and references quoted therein); $m_{\text {red }}$ is the neutralino-nucleon reduced mass. The quantities $F_{h, H}$ and $I_{h, H}$ are defined as follows:

$$
\begin{aligned}
F_{h} & =\left(-a_{1} \sin \theta_{W}+a_{2} \cos \theta_{W}\right)\left(a_{3} \sin \alpha+a_{4} \cos \alpha\right), \\
F_{H} & =\left(-a_{1} \sin \theta_{W}+a_{2} \cos \theta_{W}\right)\left(a_{3} \cos \alpha-a_{4} \sin \alpha\right), \\
I_{h, H} & =\sum_{q} k_{q}^{h, H} m_{q}\langle N|\bar{q} q| N\rangle .
\end{aligned}
$$

The matrix elements $\langle N|\bar{q} q| N\rangle$ are meant over the nucleonic state. The values adopted here for $m_{q}\langle N|\bar{q} q| N\rangle$ are the ones denoted by set 1 in Ref. [24]. We remind that uncertainties in the values of $m_{q}\langle N|\bar{q} q| N\rangle$ can give rise to an increase of the neutralino-nucleon cross section of about a factor of a few [25].

The angle $\alpha$ rotates $H_{1}^{(0)}$ and $H_{2}^{(0)}$ into $h$ and $H$, and the coefficients $k_{q}^{h, H}$ are given by 


$$
\begin{aligned}
& k_{u \text {-type }}^{h}=\cos \alpha / \sin \beta, \\
& k_{d \text {-type }}^{h}=-\sin \alpha / \cos \beta-\epsilon \cos (\alpha-\beta) \tan \beta, \\
& k_{u \text {-type }}^{H}=\sin \alpha / \sin \beta, \\
& k_{d \text {-type }}^{H}=\cos \alpha / \cos \beta-\epsilon \sin (\alpha-\beta) \tan \beta,
\end{aligned}
$$

for the up-type and down-type quarks, respectively.

In the discussion which follows we only wish to establish some correlations implied by the dependence of $\Omega_{\chi} h^{2}$ and of $\sigma_{\text {scalar }}^{\text {(nucleon) }}$ on the Higgs boson masses and the neutralino mass. For this purpose we rewrite the two previous expressions as follows:

$$
\begin{gathered}
\Omega_{\chi} h^{2} \simeq C \frac{\left[\left(2 m_{\chi}\right)^{2}-m_{A}^{2}\right]^{2}}{m_{\chi}^{2}\left[1-m_{b}^{2} / m_{\chi}^{2}\right]^{1 / 2}}, \\
\sigma_{\text {scalar }}^{\text {(nucleon) }} \simeq \frac{D}{m_{h}^{4}},
\end{gathered}
$$

with obvious definitions for $C$ and $D$. Here $m_{h}$ stands generically for the mass of the one of the two $C P$-even neutral Higgs bosons which provides the dominant contribution to $\sigma_{\text {scalar }}^{\text {(nucleon) }}$.

We now consider the case of very light neutralinos, i.e., $m_{\chi} \ll \frac{1}{2} m_{A}$. Therefore we may further simplify Eq. (8) as

$$
\Omega_{\chi} h^{2} \simeq C \frac{m_{A}^{4}}{m_{\chi}^{2}\left[1-m_{b}^{2} / m_{\chi}^{2}\right]^{1 / 2}} \text {. }
$$

The largest neutralino-nucleon scattering cross sections occur when both $m_{h}$ and $m_{A}$ are close to their experimental lower bound $\left(m_{h} \sim m_{A} \sim 90-100 \mathrm{GeV}\right)$ and $\tan \beta$ is relatively large, in which case also the couplings of Eqs. (6),(7) between neutralinos and down-type quarks through $h$ exchange are sizable [18]. In this case, from Eqs. (9) and (10) one derives the range of $\sigma_{\text {scalar }}^{\text {(nucleon) }}$ at fixed value of $m_{\chi}$ (always in the regime $\left.m_{\chi} \ll \frac{1}{2} m_{A}\right)$ :

$$
\frac{C D}{m_{\chi}^{2}\left[1-m_{b}^{2} / m_{\chi}^{2}\right]^{1 / 2}\left(\Omega_{\chi} h^{2}\right)_{\max }} \lesssim \sigma_{\text {scalar }}^{\text {(nucleon) }} \lesssim \frac{D}{m_{h, \text { min }}^{4}},
$$

where $m_{h \text {,min }}$ stands for the experimental lower bound on $m_{h}$. The lower limit to $\sigma_{\text {scalar }}^{\text {(nucleon) }}$ displayed in Eq. (11) provides a stringent lower bound on $\sigma_{\text {scalar }}^{\text {(nucleon) }}$ for very light neutralinos. This feature will show up in the numerical evaluations presented in the next section. The upper bound on $\sigma_{\text {scalar }}^{\text {(nucleon) }}$ is instead determined by the lower limit on the Higgs boson mass $m_{h}$.

By the arguments given above, it turns out that in the small mass regime $\left(m_{\chi} \ll \frac{1}{2} m_{A}\right)$ the upper bound on the relic abundance $\Omega_{\chi} h^{2} \leqslant 0.3$ establishes a constraint between the otherwise independent parameters $m_{\chi}$ and $m_{A}$ [see Eq. (10)].
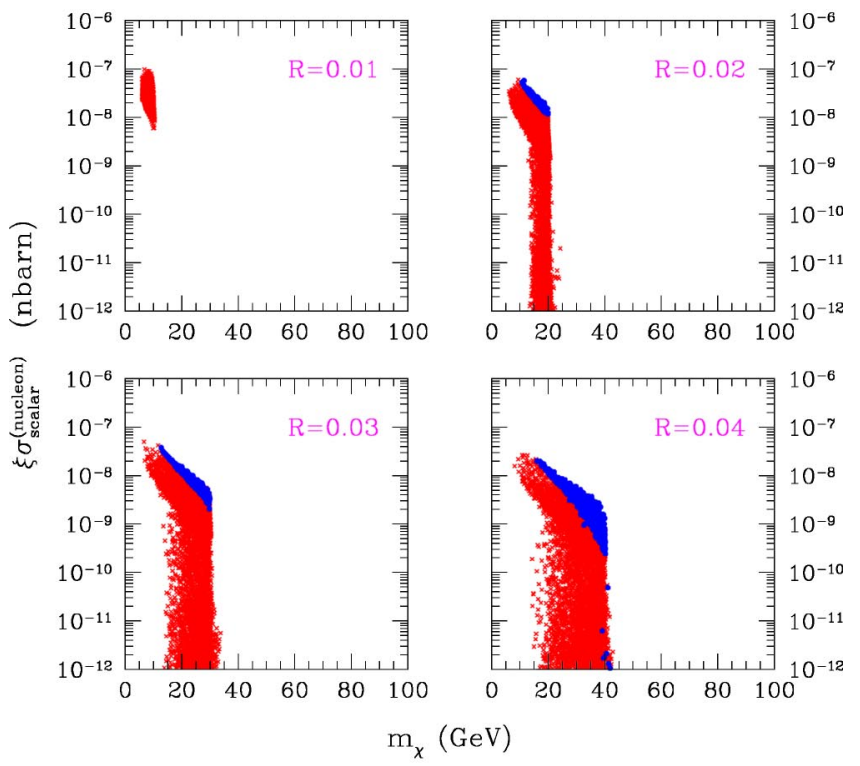

(a)
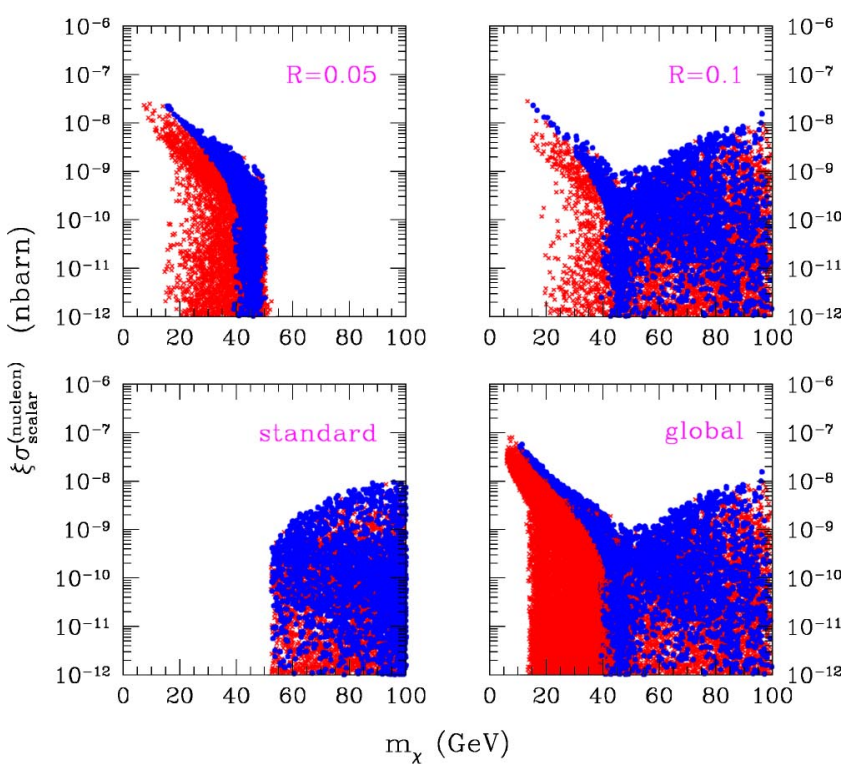

(b)

FIG. 1. (a) Scatter plots of the neutralino-nucleon cross section $\sigma_{\text {scalar }}^{\text {(nucleon }}$ times the rescaling factor $\xi$ vs the neutralino mass, for nonuniversal gaugino models with different values of the gaugino mass ratio $R=M_{1} / M_{2}: R=0.01,0.02,0.03,0.04$. Crosses denote configurations with dominant relic neutralinos $\left(0.05 \leqslant \Omega_{\chi} h^{2}\right.$ $\leqslant 0.3)$, while dots refer to subdominant neutralinos $\left(\Omega_{\chi} h^{2}\right.$ $<0.05$ ). (b) The same as in (a), for $R=0.05,0.1$, for the standard value $R=5 / 3 \tan ^{2} \theta_{W} \simeq 0.5$ and for a generic variation of $R$ in the interval $0.01-0.5$.

\section{B. Numerical results}

We turn now to the presentation of our numerical results. In Figs. 1(a),1(b) we give the scatter plots of the quantity $\xi \sigma_{\text {scalar }}^{\text {(nucleon }}$ in terms of the neutralino mass for different values of the parameter $R$. The quantity $\xi$ is defined as the ratio of the local (solar neighborhood) neutralino matter density to the total local dark matter density: $\xi \equiv \rho_{\chi} / \rho_{\text {loc }}$. In Figs. 1(a), 1 (b) we plot the quantity $\xi \sigma_{\text {scalar }}^{\text {(nucleon) }}$, rather than simply 
$\sigma_{\text {scalar }}^{\text {(nucleon) }}$, in order to include in our considerations also neutralino configurations of low relic abundance (i.e., cosmologically subdominant neutralinos). We recall that, from experimental measurements of the direct detection rates, only the product $\xi \sigma_{\text {scalar }}^{\text {(nucleon) }}$ may be extracted, and not directly $\sigma_{\text {scalar }}^{\text {(nucleon })}$. The quantity $\xi$ is derived here from the relic abundance by the usual rescaling recipe: $\xi$ $=\min \left(1, \Omega_{\chi} h^{2} /\left[\Omega h^{2}\right]_{\min }\right)$, where the minimal value of relic abundance which defines a neutralino as a dominant dark matter component has been fixed at the value $\left[\Omega h^{2}\right]_{\min }$ $=0.05 . \Omega_{\chi} h^{2}$ and $\sigma_{\text {scalar }}^{\text {(nuclen) }}$ are evaluated according to the procedures and formulas described in Refs. [24,26].

Figures 1(a),1(b) display quite remarkable properties of the light relic neutralinos from the point of view of their detectability by weakly interacting massive particle (WIMP) direct measurements. These properties are easily understandable in terms of the analytic arguments presented in the previous section. For instance, in each panel at a fixed value of $R \leqq 0.1$, there is a characteristic funnel pointing toward high values of $\xi \sigma_{\text {scalar }}^{\text {(nucleon) }}$ at small neutralino masses. This originates in the lower bound on $\sigma_{\text {scalar }}^{\text {(nucleon) }}$ reported in Eq. (11), which is effective only for very low neutralino masses (below about $15 \mathrm{GeV}$ ) and becomes more and more stringent as $m_{\chi}$ decreases. As displayed in Eq. (11), the size of this lower bound, apart from relevant supersymmetric details, is determined by the value of $\left(\Omega_{\chi} h^{2}\right)_{\max }$, which is set here at the value $\left(\Omega_{\chi} h^{2}\right)_{\max }=0.3$. It is noticeable that at very small values of $R$, for instance at $R=0.01$, all supersymmetric configurations are within the cosmologically interesting range of $\Omega_{\chi}$ (i.e. no configuration of this set is rescaled) and provide large values of $\sigma_{\text {scalar }}^{\text {(nucleon) }}$ (i.e. large detection rates).

As we increase the value of $R$, in our scan we are accessing larger values of $m_{\chi}$ : again the largest values of $\xi \sigma_{\text {scalar }}^{\text {(nucleon }}$ are dominated by Higgs exchange, for Higgs boson masses close to their lower bound of about $90 \mathrm{GeV}$. This is also true for the annihilation cross section. This approaches its pole at $m_{\chi} \sim m_{A} / 2$; therefore, the largest values of $\xi \sigma_{\text {scalar }}^{\text {(nucleon }}$ refer to subdominant neutralinos, as $m_{\chi}$ increases toward $m_{\chi} \sim 45 \mathrm{GeV}$ (which represents the pole in the annihilation cross section for the lightest possible $A$ boson). These features are clearly shown in Figs. 1(a),1(b). The panel denoted by "standard" in Fig. 1(b) refers to the usual case of universal gaugino masses: in this case the neutralino mass is bounded from below at about $50 \mathrm{GeV}$, and therefore all the interesting low neutralino-mass sector is precluded. The last panel in Fig. 1(b) (denoted by "global") shows our results for $R$ varied in the interval 0.01-0.5: the funnel at low masses and the effect of the $A$ pole in the annihilation cross section are clearly visible.

We recall that, for each panel at fixed $R$, the lower value of the neutralino mass is a consequence of the experimental bound on the chargino mass, which in turn fixes a lower bound on $M_{1}=R \times M_{2}$. The upper value on the neutralino mass for each panel is a mere consequence of the fact that we scan the $M_{2}$ parameter up to $1 \mathrm{TeV}$.

The detailed connection among the values of $\sigma_{\text {scalar }}^{\text {(nucleon }}$ and those of $\Omega_{\chi} h^{2}$ is given in Fig. 2. The strong correlation between $\sigma_{\text {scalar }}^{\text {(nucen) }}$ and $\Omega_{\chi} h^{2}$ displayed for $R=0.01$ reflects the
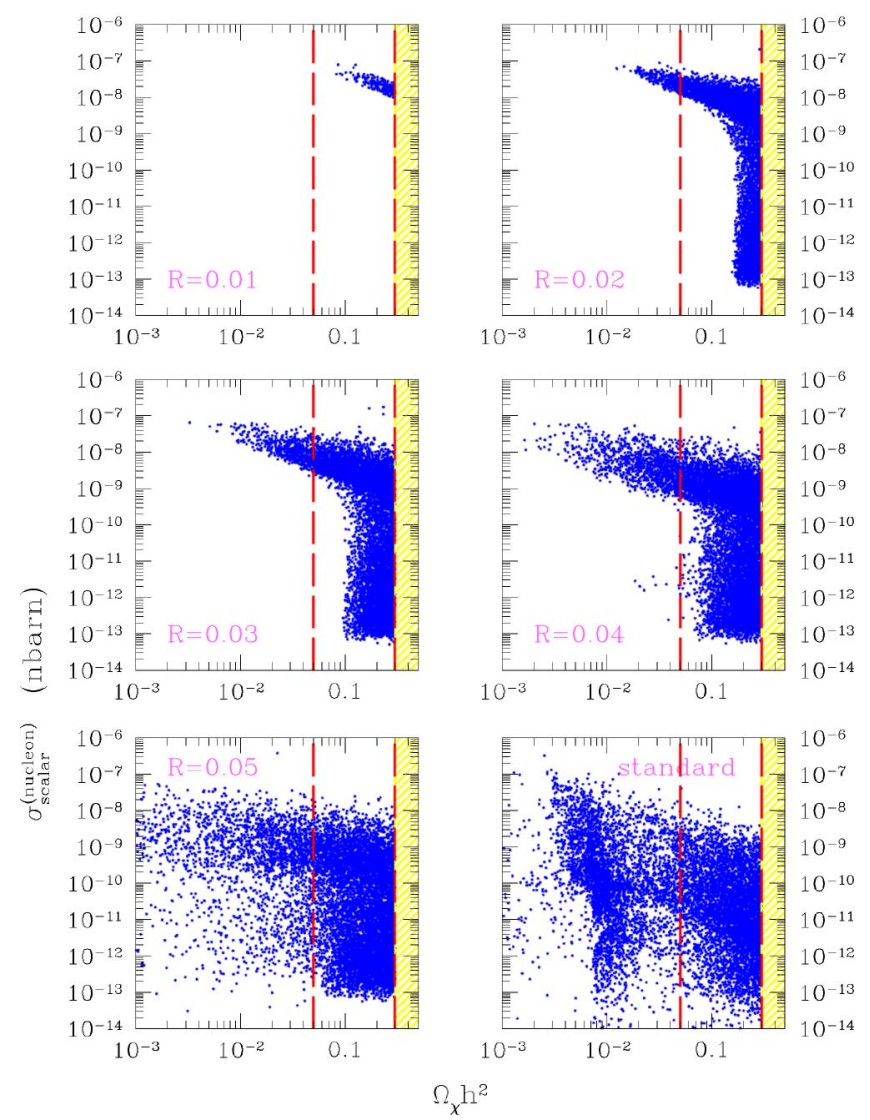

FIG. 2. Scatter plots of the neutralino-nucleon cross section $\sigma_{\text {scalar }}^{\text {(nucleon }}$ vs the neutralino relic abundace $\Omega_{\chi} h^{2}$, for $R$ $=0.01,0.02,0.03,0.04,0.05$ and for the standard value $R$ $=5 / 3 \tan ^{2} \theta_{W} \simeq 0.5$.

properties of the funnel previously discussed in connection with Fig. 1(a). All the configurations refer to large values of $\Omega_{\chi} h^{2}$ : actually, it is the upper bound on the neutralino relic abundance which determines the strong bound on the allowed configurations. By changing $R$ from 0.01 to larger values, we observe that the ensuing increase in $m_{\chi}$ shifts the configurations of largest $\sigma_{\text {scalar }}^{\text {(nucleon }}$ toward lower values of relic abundance, as expected from the analytical considerations of the previous section. From this figure we see that a fraction of the largest values of the quantity $\xi \sigma_{\text {scalar }}^{\text {(nucleon) }}$ refer to dominant neutralinos, while another fraction refers to slightly subdominant neutralinos: $0.01 \leq \Omega_{\chi} h^{2} \leq 0.05$. Configurations with $\Omega_{\chi} h^{2}<0.01$, even providing the largest values of the scattering cross section (see, for instance, the panel at $R=0.04$ in Fig. 2) suffer from a severe rescaling factor $\xi$ which somehow reduces their detectability.

The fact that for small values of $R$ the scattering and neutralino-neutralino annihilation cross sections are dominated by Higgs exchange is a consequence of two facts: the relatively small values for the lower bounds on $m_{h}$ and $m_{A}$ and the neutralino composition, which, even though dominated by the $B$-ino component, nevertheless possesses a nonnegligible Higgsino contribution allowing the neutralino to efficiently couple with the Higgs fields.

Figure 3 shows that for small values of $R$ (small $m_{\chi}$ ) the neutralino-neutralino annihilation cross section is indeed 


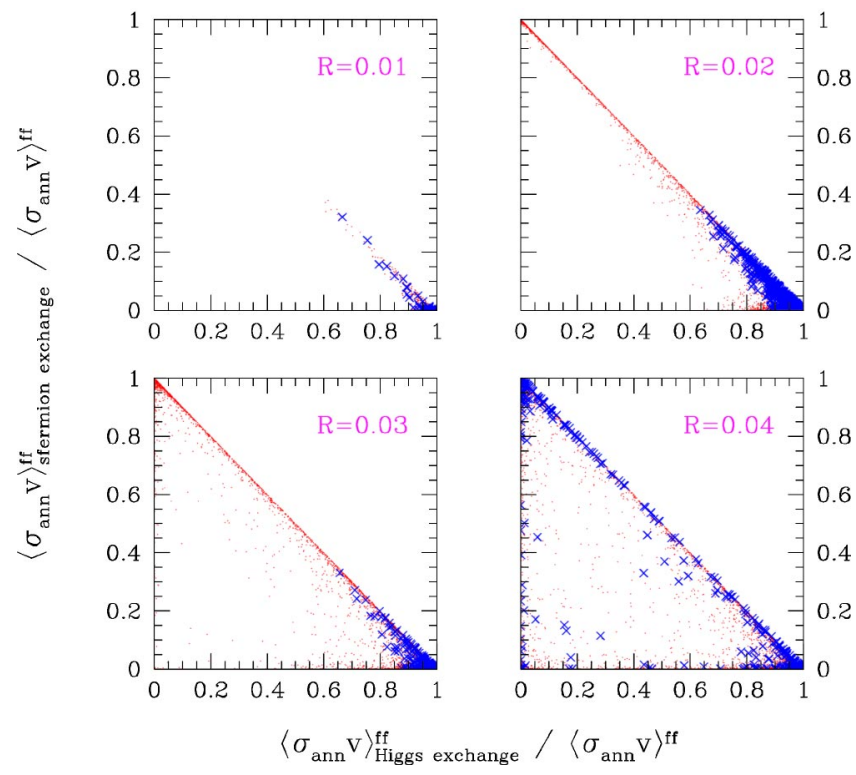

FIG. 3. Scatter plots of the fractional amount of the neutralino pair-annihilation cross section due to sfermion exchange vs Higgs boson exchange, for $R=0.01,0.02,0.03,0.04$. Crosses denote configuration for which the neutralino-nucleon scattering cross section $\sigma_{\text {scalar }}^{\text {(nucleon) }}$ is larger than $10^{-8}$ nbarn.

dominated by Higgs-exchange diagrams, especially for the largest values of $\sigma_{\text {scalar }}^{\text {(nucleon) }}$. The first panel of Fig. 3, which refers to $R=0.01$, clearly shows that the annihilation cross section is strongly dominated by Higgs exchange. For $R$ $=0.02$ the annihilation cross section can be either dominated by Higgs or sfermion exchange: however, the configurations which provide values of $\sigma_{\text {scalar }}^{\text {(nucleon }}$ in excess of $10^{-8}$ nbarn (denoted by crosses) show a clear Higgs dominance in the annihilation cross section. These features are progressively lost when $R$ increases: the annihilation cross section may be dominated by $Z$ exchange (which, by coincidence, has its pole also at about $45 \mathrm{GeV}$ ).

Finally, Fig. 4 shows that for low values of $R$, the neutralino composition is dominated by the $B$-ino component, but a deviation from a pure $B$-ino composition is present and is mainly due to a mixture of $\widetilde{B}$ with $\widetilde{H}_{1}^{0}$. The two composition parameters $a_{1}^{2}$ and $a_{3}^{2}$ remain aligned along the $a_{1}^{2}+a_{3}^{2}$ $=1$ diagonal line up to $R \sim 0.05$, with a clear dominance (above 70\%) in $B$-ino. For larger values of $R$ the correlation between $a_{1}^{2}$ and $a_{3}^{2}$ starts to deviate from the diagonal line, a fact that indicates how the two other components are becoming important (it is mainly $a_{4}$ which sets up). The panel at $R=0.1$ shows that the $B$-ino component is usually large, but a sizable mixture starts occurring. The last panel in Fig. 4 recalls the situation for the standard case of universal gaugino masses, where the neutralino may be any mixture of its component fields.

\section{CONCLUSIONS}

In the present paper we have focussed our attention on relic neutralinos of light masses: $m_{\chi} \lesssim 45 \mathrm{GeV}$, which are allowed in supersymmetric models where no unification of gaugino masses is assumed. We have shown that these neu-
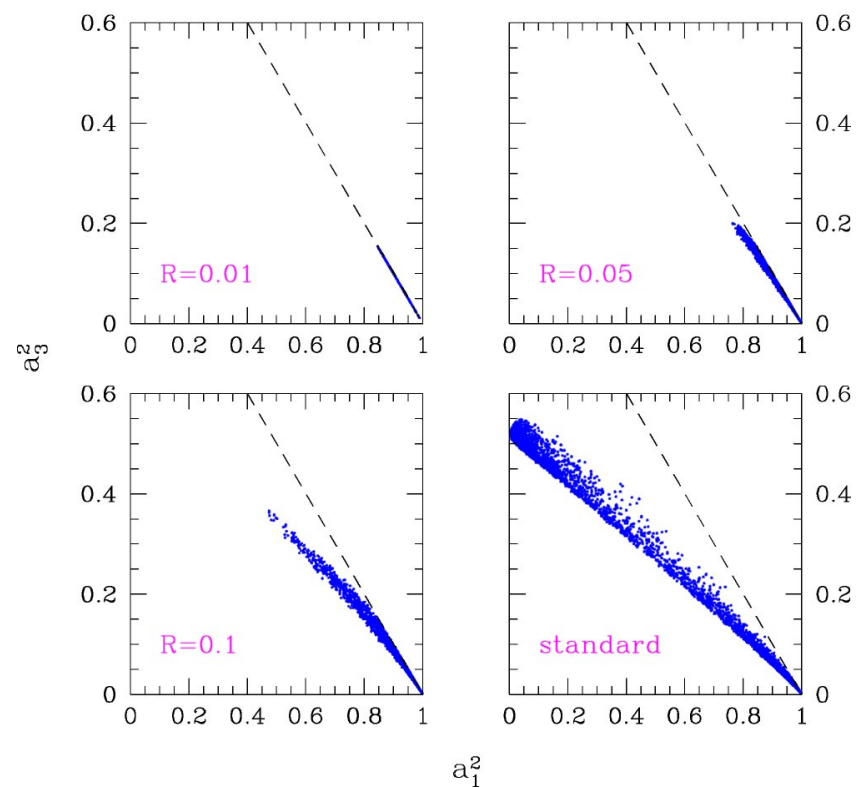

FIG. 4. Scatter plots of the neutralino composition in terms of $\widetilde{B}$ $\left(a_{1}\right)$ and of $\widetilde{H}_{1}^{\circ}\left(a_{3}\right)$ for $R=0.01,0.05,0.1$ and for the standard value $R=5 / 3 \tan ^{2} \theta_{W} \simeq 0.5$. The dashed lines denote the line where $a_{1}^{2}$ $+a_{3}^{2}=1$.

tralinos may have elastic cross sections off nucleons which go up to $\sigma_{\text {scalar }}^{\text {(nucleon) }} \sim 10^{-7}$ nbarn, with a relic abundance of cosmological interest: $0.05 \leqq \Omega_{\chi} h^{2} \leq 0.3$.

The present upper limits to $\xi \sigma_{\text {scalar }}^{\text {(nucleon) }}$ provided by WIMP direct detection experiments [27-30] do not constrain the supersymmetric configurations for the light neutralinos considered here. This is especially true once the relevant uncertainties (mainly related to the form and parameters of the WIMP galactic distribution function [31] and to the quenching factors for bolometric detectors) are taken into account. The CDMS upper bound [29] could concern a small fraction of supersymmetric configurations in the range around 15 $\mathrm{GeV}$, though very marginally, if the uncertainties on astrophysical quantities are considered. Moreover, the CDMS bound needs a confirmation by a further running in a deepunderground site, as planned by the Collaboration.

The small-mass neutralino configurations analyzed in the present paper are accessible to experiments of direct detection with a low-energy threshold and a high sensitivity. An experiment of this type is the DAMA experiment with a mass of $\simeq 100 \mathrm{~kg}$ of $\mathrm{NaI}(\mathrm{Tl})$, whose results after a 4-years running show an annual-modulation effect at a $4 \sigma$ C.L. which does not appear to be related to any possible source of systematics [32]. The DAMA experiment, with its high sensitivity, is potentially good to investigate also the relic neutralinos considered in the present paper.

\section{ACKNOWLEDGMENTS}

We gratefully acknowledge financial support provided by Research Grants of the Italian Ministero dell'Istruzione, dell'Università e della Ricerca (MIUR) and of the Università di Torino within the Astroparticle Physics Project. 
[1] J. Ellis, K. Enqvist, D. V. Nanopoulos, and K. Tamvakis, Phys. Lett. 155B, 381 (1985); M. Drees, ibid. 158B, 409 (1985); Phys. Rev. D 33, 1468 (1986).

[2] M. Drees and X. Tata, Phys. Rev. D 43, 2971 (1991).

[3] K. Griest and L. Roszkowski, Phys. Rev. D 46, 3309 (1992).

[4] S. Mizuta, D. Ng, and M. Yamaguchi, Phys. Lett. B 300, 96 (1993).

[5] A. Gabutti, M. Olechowski, S. Cooper, S. Pokorski, and L. Stodolsky, Astropart. Phys. 6, 1 (1996).

[6] G. Bélanger, F. Boudjema, F. Donato, R. Godbole, and S. Rosier-Lees, Nucl. Phys. B581, 3 (2000).

[7] A. Corsetti and P. Nath, Phys. Rev. D 64, 125010 (2001).

[8] R. Arnowitt, B. Dutta, and Y. Santoso, hep-ph/0010244.

[9] D. G. Cerdeño, S. Khalil, and C. Muñoz, hep-ph/0105180.

[10] V. A. Bednyakov and H. V. Klapdor-Kleingrothaus, Phys. Rev. D 63, 095005 (2001).

[11] H. Baer, C. Balazs, A. Belyaev, R. Dermisek, A. Mafi, and A. Mustfayev, J. High Energy Phys. 05, 061 (2002).

[12] V. Bertin, E. Nezri, and J. Orloff, hep-ph/0210034.

[13] A. Birkedal-Hansen and B. D. Nelson, hep-ph/0211071; Phys. Rev. D 64, 015008 (2001).

[14] A. Bottino, F. Donato, N. Fornengo, and S. Scopel, Phys. Lett. B 423, 109 (1998); Phys. Rev. D 59, 095003 (1999); 59, 095004 (1999); 62, 056006 (2000).

[15] ALEPH Collaboration, A. Colaleo, talk at SUSY'01, 2001, Dubna, Russia; DELPHI Collaboration, J. Abdallah et al., DELPHI 2001-085 CONF 513, 2001.

[16] T. Affolder et al., Phys. Rev. Lett. 86, 4472 (2001).

[17] CLEO Collaboration, S. Ahmed et al., CONF 99/10, hep-ex/9908022; ALEPH Collaboration, R. Barate et al., Phys. Lett. B 429, 169 (1998); Belle Collaboration, K. Abe et al., ibid. 511, 151 (2001).

[18] A. Bottino, N. Fornengo, and S. Scopel, Nucl. Phys. B608, 461 (2001).

[19] A. Bottino, F. Donato, N. Fornengo, and S. Scopel, Phys. Rev. D 63, 125003 (2001).

[20] S. Ambrosanio, A. Dedes, S. Heinemeyer, S. Su, and G. Wei- glein, Nucl. Phys. B624, 3 (2002).

[21] Muon $(g-2)$ Collaboration, G. W. Bennett et al., Phys. Rev. Lett. 89, 101804 (2002); 89, 129903(E) (2002).

[22] M. Davier, S. Eidelman, A. Höcker, and Z. Zhang, hep-ph/0208177.

[23] K. Hagiwara, A. D. Martin, D. Nomura, and T. Teubner, hep-ph/0209187.

[24] A. Bottino, F. Donato, N. Fornengo, and S. Scopel, Astropart. Phys. 13, 215 (2000).

[25] A. Bottino, F. Donato, N. Fornengo, and S. Scopel, Astropart. Phys. 18, 205 (2002).

[26] A. Bottino, V. de Alfaro, N. Fornengo, G. Mignola, and M. Pignone, Astropart. Phys. 2, 67 (1994).

[27] For a summary on WIMP direct detection experiments, see, for instance, A. Morales, Nucl. Phys. B (Proc. Suppl.) 110, 39 (2002).

[28] DAMA/NaI Collaboration, R. Bernabei et al., Phys. Lett. B 389, 757 (1996).

[29] CDMS Collaboration, D. Abrams et al., Phys. Rev. D 66, 122003 (2002).

[30] A. Benoit et al., Phys. Lett. B 545, 43 (2002).

[31] P. Belli, R. Cerulli, N. Fornengo, and S. Scopel, Phys. Rev. D 66, 043503 (2002).

[32] DAMA/NaI Collaboration, R. Bernabei et al., Phys. Lett. B 480, 23 (2000); Eur. Phys. J. C 18, 283 (2000).

[33] G. Bélanger, F. Boudjema, A. Pukhov, and S. Rosier-Lees, hep-ph/0212227.

[34] D. Hooper and T. Plehn, hep-ph/0212226.

[35] This is at variance with the results of Refs. [33,34], where a lower limit on the neutralino mass of $12 \mathrm{GeV}$ [33] and $18 \mathrm{GeV}$ [34] has been deduced. Notice that the authors of Refs. [33,34] consider only the limit of very large $m_{A}$, which strongly suppresses processes which involve $A$ exchange, in particular the neutralino annihilation cross section. On the contrary, we are considering also the light Higgs sector, which is effective in reducing the value of the neutralino relic abundance and therefore in allowing lighter neutralinos [see Eq. (10)]. 